ALEA, Lat. Am. J. Probab. Math. Stat. 16, 1201-1211 (2019)

DOI: 10.30757/ALEA.v16-45

\title{
On equalities of sigma-finite measures constructed from discrete recurrent Markov chains
}

\author{
Joseph Najnudel and Ju-Yi Yen \\ School of Mathematics, \\ University of Bristol, \\ Bristol, BS8 1QU, UK \\ E-mail address: joseph.najnudel@bristol.ac.uk \\ Department of Mathematical Sciences, \\ University of Cincinnati, \\ Cincinnati, OH 45221-0025, USA \\ E-mail address: ju-yi.yen@uc.edu
}

\begin{abstract}
In Salminen et al. (2015), Salminen, Yen and Yor have proven several equalities of sigma-finite measures involving the law of one-dimensional recurrent diffusions and some of its $h$-transforms. In the present article, we show that similar equalities hold in the setting of irreducible and recurrent discrete Markov chains.
\end{abstract}

\section{Introduction}

In Salminen et al. (2015), the authors find some relationships between different sigma-finite measures associated to recurrent one-dimensional diffusions and their excursions. More precisely, let $X$ be a one-dimensional diffusion which is regular, recurrent, taking values on $\mathbb{R}_{+}$with 0 as an instantaneously reflecting boundary point (see Salminen et al., 2007 for detailed setting), the authors define another process $X^{\uparrow}$ using the $h$-transform of the process $X$ killed when it hits zero; the process $X^{\uparrow}$ can be seen as $X$ conditioned to stay positive. For example, if $X$ is a reflected Brownian motion, $X^{\uparrow}$ is a Bessel process of dimension three. The notion of local times, excursions, and Itô measure are well-defined in this setting. One can then define the following measures:

- $\mathbb{P}_{0}$ is the law of the diffusion $X$, starting at zero, $\mathbb{P}_{0}^{t}$ and $\mathbb{P}_{0}^{\tau \ell}$ correspond the law of the same process, stopped respectively at time $t$ and at the inverse local time $\tau_{\ell}$.

- $\mathbb{P}_{0}^{\uparrow}$ is the law of the diffusion $X^{\uparrow}$ starting at zero.

Received by the editors December 21th, 2018; accepted October 30th, 2019.

2010 Mathematics Subject Classification. 60J10, 60J45, 60J50, 60J55.

Key words and phrases. Markov chain, sigma-finite measure, hitting time, local time, harmonic function. 
- $\mathbb{P}_{0, u, 0}$ is the law of the bridge of $X$ with length $u$, starting and ending at zero.

- $\mathbf{n}$ is the Itô measure of the excursion of $X, \mathbf{n}^{t}$ is the image of $\mathbf{n}$ by restricting the trajectories to the interval $[0, t]$.

All these measures are very closely related. In Salminen et al. (2015), the authors prove the following equalities of sigma-finite measures:

$$
\begin{gathered}
\int_{0}^{\infty} \mathbb{P}_{0}^{t} d t=\int_{0}^{\infty} \mathbb{P}_{0}^{\tau_{\ell}} d \ell \circ \int_{0}^{\infty} d u \mathbf{n}^{u}(\cdot ; u<\zeta), \\
\int_{0}^{\infty} \mathbb{P}_{0}^{\tau_{\ell}} d \ell=\int_{0}^{\infty} d u p_{u}(0,0) \mathbb{P}_{0, u, 0}, \\
\mathbf{n}\left(G_{t} ; t<\zeta\right)=\mathbb{E}_{\mathbb{P}_{0}^{\uparrow}}\left[G_{t} \frac{1}{S\left(\omega_{t}\right)}\right] \\
\int_{0}^{\infty} \mathbf{n}^{t}(\cdot ; t<\zeta)=\int_{0}^{\infty} m(d a)\left(\mathbb{P}_{0}^{\uparrow}\right)^{\gamma_{a}}
\end{gathered}
$$

Here, the integrals correspond to disintegration of sigma-finite measures, the symbol o represents concatenation of trajectories, $\zeta$ is the life-time of the excursions, $S$ is the scale function and $m$ is the speed measure of $X, p_{u}(0,0)$ is the corresponding transition density with respect to the measure $m, G_{t}$ is a non-negative functional, measurable with respect to the $\sigma$-algebra generated by the canonical trajectory $\omega$ up to time $t$, and $\gamma_{a}$ denotes the last hitting time of $a$.

The results in Salminen et al. (2015) are generalizations of the same results in the case of the Brownian motion and the three-dimensional Bessel process, proven in Biane and Yor (1987) in this particular case. These equalities are directly related to different ways of splitting the trajectories of the processes $X$ and $X^{\uparrow}$ at different random times. In order to see this more clearly, we develop a similar setting adapted to discrete recurrent Markov chains, which have the advantage that we can consider the measure of individual trajectories. The setting will be the same as in the last chapter of Najnudel et al. (2009) and in Najnudel (2015), where some sigma-finite measures related to the law of the Markov chains are considered.

An analog of the two first equalities cited above is proven in Section 2. The two last equalities have a priori no immediate analog in the setting of Markov chains, since they involve the probability measure $\mathbb{P}_{0}^{\uparrow}$. In Section 3 , we use a particular construction of transient Markov chain in order to solve this issue. We then prove an analog of the third equality above, and of the fourth equality in the setting of the simple random walk on $\mathbb{Z}$ or $\mathbb{Z}^{2}$.

\section{Presentation of the setting and proof of two equalities of sigma-finite measures}

We consider a countable state space $E,\left(X_{n}\right)_{n \geq 0}$ the canonical process defined on $E^{\mathbb{N}_{0}}\left(\mathbb{N}_{0}:=\{0,1,2, \ldots\}\right),\left(\mathcal{F}_{n}\right)_{n \geq 0}$ the natural filtration of $\left(X_{n}\right)_{n \geq 0}$ and $\mathcal{F}_{\infty}$ the $\sigma$-algebra generated by $\mathcal{F}_{n}$ for $n \geq 0$. We define a family $\left(\mathbb{P}_{x}\right)_{x \in E}$ of probability measures on the filtered measurable space $\left(E^{\mathbb{N}_{0}}, \mathcal{F}_{\infty},\left(\mathcal{F}_{n}\right)_{n>0}\right)$ corresponding to a Markov chain, i.e. there exists a family $\left(p_{y, z}\right)_{y, z \in E}$ of elements of $[0,1]$ such that 
for all $k \geq 0, x_{0}, \ldots x_{k} \in E$,

$$
\mathbb{P}_{x}\left(X_{0}=x_{0}, X_{1}=x_{1}, \ldots, X_{k}=x_{k}\right)=\mathbb{1}_{x_{0}=x} \prod_{j=1}^{k} p_{x_{j-1}, x_{j}} .
$$

We will denote by $\mathbb{E}_{x}$ the expectation under $\mathbb{P}_{x}$. We assume:

- For all $x \in E, p_{x, y}>0$ for finitely many $y \in E$.

- The Markov chain associated to the probability measures $\left(\mathbb{P}_{x}\right)_{x \in E}$ is irreducible and recurrent.

Let us now fix a point $x_{0} \in E$. By the recurrence of the Markov chain, the canonical trajectory almost surely hits $x_{0}$ infinitely many times under $\mathbb{P}_{x}$, for all $x \in E$. For $k \geq 1$, we define $\tau_{k}$ as the $k$-th strictly positive value of $n$ such that $X_{n}=x_{0}$ (inverse local time at $x_{0}$ ). Under $\mathbb{P}_{x_{0}}$, if we stop the canonical trajectory at $\tau_{1}$, the first return time at $x_{0}$, we get almost surely a finite trajectory $\mathbf{e}_{1}:=\left(X_{0}, X_{1}, \ldots, X_{\tau_{1}}\right)$, such that

$$
\mathbb{P}_{x_{0}}\left(\tau_{1}=k, \mathbf{e}_{1}=\left(x_{0}, x_{1}, \ldots, x_{k}\right)\right)=\left(\prod_{j=1}^{k} p_{x_{j-1}, x_{j}}\right) \mathbb{1}_{x_{1}, \ldots, x_{k-1} \neq x_{0}, x_{k}=x_{0}} .
$$

The trajectory $\mathbf{e}_{1}$ is the first excursion, starting and ending at $x_{0}$, of the canonical process $\left(X_{k}\right)_{k \geq 0}$ : the formula just above gives its probability distribution under $\mathbb{P}_{x_{0}}$.

Example 2.1. The law of a standard random walk on $\mathbb{Z}$ corresponds to the following distribution for the excursions starting from 0: a trajectory starting at zero, ending at zero and never hitting zero in between has probability $2^{-k}$ when it stops at time $k$.

We can also notice that the distribution $\mathbb{P}_{x_{0}}$ can be recovered from the distribution of $\mathbf{e}_{1}$, by using the strong Markov property: under $\mathbb{P}_{x_{0}}$, the canonical process has the same law as the concatenation of i.i.d. excursions distributed as $\mathbf{e}_{1}$. The results we will prove in this article are related to this fact.

For a deterministic or random time $T$, and for $x \in E$, we denote by $\mathbb{P}_{x}^{T}$ the law of the Markov chain starting at $x$, the trajectory being stopped at time $T$. Moreover, if $\mathbb{Q}_{1}$ and $\mathbb{Q}_{2}$ are two measures (not necessarily of total mass equal to 1 ), respectively on $E^{\ell+1}$ and $E^{\ell^{\prime}+1}$ for some nonnegative integers $\ell$ and $\ell^{\prime}$, we define the measure $\mathbb{Q}_{1} \circ \mathbb{Q}_{2}$ as the image of the product measure $\mathbb{Q}_{1} \otimes \mathbb{Q}_{2}$ by the map

$$
\left(\left(Y_{0}, \ldots, Y_{\ell}\right),\left(Z_{0}, \ldots, Z_{\ell^{\prime}}\right)\right) \mapsto\left(Y_{0}, \ldots, Y_{\ell}, Z_{1}, \ldots, Z_{\ell^{\prime}}\right) .
$$

In the case where $\mathbb{Q}_{1}$ and $\mathbb{Q}_{2}$ are probability measures and $Y_{\ell}=Z_{0}$ almost surely under $\mathbb{Q}_{1} \otimes \mathbb{Q}_{2}$, the probability measure $\mathbb{Q}_{1} \circ \mathbb{Q}_{2}$ is the law of the concatenation of two independent paths, respectively following $\mathbb{Q}_{1}$ and $\mathbb{Q}_{2}$. With this notation, we have the following equality of $\sigma$-finite measures, where by convention we set $\tau_{0}:=0$ :

Proposition 2.2. It holds:

$$
\sum_{n=0}^{\infty} \mathbb{P}_{x_{0}}^{n}=\left(\sum_{k=0}^{\infty} \mathbb{P}_{x_{0}}^{\tau_{k}}\right) \circ\left(\sum_{m=0}^{\infty} \mathbf{p}_{m}\right),
$$

where $\mathbf{p}_{m}$ is the finite measure on $E^{m+1}$ given by

$$
\mathbf{p}_{m}\left(x_{0}, \ldots, x_{m}\right)=\mathbb{P}_{x_{0}}\left[X_{0}=x_{0}, \ldots, X_{m}=x_{m}, \tau_{1}>m\right] .
$$


Proof: Almost every trajectory, under the measures in both sides of the equality, starts at $x_{0}$. Let $\left(x_{0}, \ldots, x_{n}\right)$ be such a trajectory. We assume that it returns $k$ times to $x_{0}$, the last hitting time being $r$ (then $x_{r}=x_{0}$ ). If $k=0$, we set $r:=0$.

Under the $\sigma$-finite measure on the left-hand side, the trajectory $\left(x_{0}, \ldots, x_{n}\right)$ has measure $p_{x_{0}, x_{1}} \ldots p_{x_{n-1} x_{n}}$.

Under the first measure in the right-hand side, the trajectories start and end at $x_{0}$, and under the second measure in the right-hand side, the trajectories start at $x_{0}$ and then never hit $x_{0}$ again (we necessary stop the excursions strictly before their last step). Hence, the only way to split the trajectory $\left(x_{0}, \ldots, x_{n}\right)$ into a trajectory with non-zero first measure and a trajectory with non-zero second measure is at time $r$. By considering the number of hitting times of $x_{0}$ of the trajectory $\left(x_{0}, \ldots, x_{r}\right)$, and the number of steps of the trajectory $\left(x_{r}, \ldots, x_{n}\right)$, we deduce that the righthand side measure of $\left(x_{0}, \ldots, x_{n}\right)$ is

$$
\mathbb{P}_{x_{0}}^{\tau_{k}}\left(x_{0}, \ldots, x_{r}\right) \cdot \mathbf{p}_{n-r}\left(x_{r}, \ldots, x_{n}\right) .
$$

Now, since $r$ is the $k$-th return time at $x_{0}$ of $\left(x_{0}, \ldots, x_{n}\right)$, we have

$$
\mathbb{P}_{x_{0}}^{\tau_{k}}\left(x_{0}, \ldots, x_{r}\right)=\mathbb{P}_{x_{0}}\left(X_{0}=x_{0}, X_{1}=x_{1}, \ldots, X_{r}=x_{r}\right)=\prod_{j=1}^{r} p_{x_{j-1}, x_{j}} .
$$

On the other hand, we have

$$
\mathbf{p}_{n-r}\left(x_{r}, \ldots, x_{n}\right)=\mathbb{P}_{x_{0}}\left(X_{0}=x_{r}, \ldots, X_{n-r}=x_{n}, \tau_{1}>n\right) .
$$

Now, if $X_{j}=x_{r+j}$ for $0 \leq j \leq n-p$, necessarily $\tau_{1}>n$ since $x_{s} \neq x_{0}$ for all $s$ between $r+1$ and $n$. Hence,

$$
\mathbf{p}_{n-r}\left(x_{r}, \ldots, x_{n}\right)=\mathbb{P}_{x_{0}}\left(X_{0}=x_{r}, \ldots, X_{n-r}=x_{n}\right)=\prod_{j=r+1}^{n} p_{x_{j-1}, x_{j}} .
$$

The right-hand side measure of $\left(x_{0}, \ldots, x_{n}\right)$ is then

$$
\left(\prod_{j=1}^{r} p_{x_{j-1}, x_{j}}\right)\left(\prod_{j=r+1}^{n} p_{x_{j-1}, x_{j}}\right)=\prod_{j=1}^{n} p_{x_{j-1}, x_{j}} .
$$

Let us now denote by $\mathbb{P}_{x_{0}, x_{0}}^{(n)}$ the law of the bridge of the Markov process from $x_{0}$ to $x_{0}$, with duration $n$, i.e. the law of $\left(X_{0}, \ldots, X_{n}\right)$ under $\mathbb{P}_{x_{0}}$, conditioned by the event $X_{n}=x_{0}$. If this event has probability zero, we take for $\mathbb{P}_{x_{0}, x_{0}}^{(n)}$ any probability measure on the trajectories indexed by $\{0, \ldots, n\}$, for example the Dirac measure at $\left(x_{0}, \ldots, x_{0}\right)$. We denote by $p_{x, y}^{(n)}$ the probability that $X_{n}=y$, under $\mathbb{P}_{x}$ : for example, $p_{x, y}^{(1)}=p_{x, y}$. We then have the following result:

Proposition 2.3. One has

$$
\sum_{k=0}^{\infty} \mathbb{P}_{x_{0}}^{\left(\tau_{k}\right)}=\sum_{n=0}^{\infty} p_{x_{0}, x_{0}}^{(n)} \mathbb{P}_{x_{0}, x_{0}}^{(n)} .
$$

Proof: Almost everywhere under the two measures, the trajectories start and stop at $x_{0}$. Let $\left(x_{0}, \ldots, x_{n}\right)$ be such a trajectory (and then $x_{n}=x_{0}$ ). Let $k$ be the 
number of its returns to $x_{0}$. The left-hand side measure of this path is

$$
\begin{aligned}
\mathbb{P}_{x_{0}}^{\left(\tau_{k}\right)}\left(x_{0}, \ldots, x_{n}\right) & =\mathbb{P}_{x_{0}}\left(X_{0}=x_{0}, \ldots, X_{n}=x_{n}, \tau_{k}=n\right) \\
& =\mathbb{P}_{x_{0}}\left(X_{0}=x_{0}, \ldots, X_{n}=x_{n}\right),
\end{aligned}
$$

the last equality coming from the fact that $X_{0}=x_{0}, \ldots, X_{n}=x_{n}$ implies $\tau_{k}=n$, since $x_{n}=x_{0}$, and $\left(x_{0}, \ldots, x_{n}\right)$ has $k$ return times at $x_{0}$.

On the other hand, in the equality of the proposition, the right-hand side measure of the trajectory $\left(x_{0}, \ldots, x_{n}\right)$ is equal to

$$
\begin{aligned}
& p_{x_{0}, x_{0}}^{(n)} \mathbb{P}_{x_{0}, x_{0}}^{(n)}\left(x_{0}, x_{1}, \ldots, x_{n}\right) \\
& =\mathbb{P}_{x_{0}}\left(X_{n}=x_{0}\right) \mathbb{P}_{x_{0}}\left(X_{0}=x_{0}, \ldots, X_{n-1}=x_{n-1}, X_{n}=x_{n} \mid X_{n}=x_{0}\right)
\end{aligned}
$$

if $\mathbb{P}_{x_{0}}\left(X_{n}=x_{0}\right)>0$, since under $\mathbb{P}_{x_{0}, x_{0}}^{(n)},\left(X_{0}, \ldots, X_{n}\right)$ is a bridge of the Markov process from $x_{0}$ to $x_{0}$, with duration $n$.

Since we consider trajectories such that $x_{n}=x_{0}$, we deduce, for $\mathbb{P}_{x_{0}}\left(X_{n}=x_{0}\right)>$ 0 , that

$$
\begin{aligned}
& p_{x_{0}, x_{0}}^{(n)} \mathbb{P}_{x_{0}, x_{0}}^{(n)}\left(x_{0}, x_{1}, \ldots, x_{n}\right) \\
& =\mathbb{P}_{x_{0}}\left(X_{n}=x_{n}\right) \mathbb{P}_{x_{0}}\left(X_{0}=x_{0}, \ldots, X_{n-1}=x_{n-1}, X_{n}=x_{n} \mid X_{n}=x_{n}\right) \\
& =\mathbb{P}_{x_{0}}\left(X_{0}=x_{0}, \ldots, X_{n-1}=x_{n-1}, X_{n}=x_{n}\right) .
\end{aligned}
$$

If $\mathbb{P}_{x_{0}}\left(X_{n}=x_{0}\right)=0$, we have $p_{x_{0}, x_{0}}^{(n)}=0$ and

$$
\begin{aligned}
& \mathbb{P}_{x_{0}}\left(X_{0}=x_{0}, \ldots, X_{n-1}=x_{n-1}, X_{n}=x_{n}\right) \\
& \leq \mathbb{P}_{x_{0}}\left(X_{n}=x_{n}\right)=\mathbb{P}_{x_{0}}\left(X_{n}=x_{0}\right)=0 .
\end{aligned}
$$

which implies that the equality

$$
p_{x_{0}, x_{0}}^{(n)} \mathbb{P}_{x_{0}, x_{0}}^{(n)}\left(x_{0}, x_{1}, \ldots, x_{n}\right)=\mathbb{P}_{x_{0}}\left(X_{0}=x_{0}, \ldots, X_{n-1}=x_{n-1}, X_{n}=x_{n}\right)
$$

remains true, and then we always have

$$
p_{x_{0}, x_{0}}^{(n)} \mathbb{P}_{x_{0}, x_{0}}^{(n)}\left(x_{0}, x_{1}, \ldots, x_{n}\right)=\mathbb{P}_{x_{0}}^{\left(\tau_{k}\right)}\left(x_{0}, x_{1} \ldots, x_{n}\right),
$$

if $\left(x_{0}, \ldots, x_{n}\right)$ is a trajectory with $k$ returns to $x_{0}$, the last one being at time $n$.

\section{Other equalities involving measures on transient trajectories}

From the probability distribution $\mathbb{P}_{x_{0}}$ we can construct, by using $h$-transforms, other measures under which the canonical process is transient. A similar construction is detailed in Najnudel (2015). We let $\varphi$ be a function from $E$ to $\mathbb{R}_{+}$, not identically zero, which vanishes at $x_{0}$ and is harmonic at all other points: for all $x \neq x_{0}$

$$
\varphi(x)=\sum_{y \in E} p_{x, y} \varphi(y) .
$$

We also normalize $\varphi$ in such a way that

$$
\mathbb{E}_{x_{0}}\left[\varphi\left(X_{1}\right)\right]=\sum_{y \in E} p_{x_{0}, x} \varphi(x)=1 .
$$

It is easy to check that under $\mathbb{P}_{x_{0}},\left(\varphi\left(X_{n \wedge \tau_{1}}\right)\right)_{n \geq 1}$ is a $\left(\mathcal{F}_{n}\right)_{n \geq 1}$-martingale, whose expectation is equal to 1 . Hence, there exists a probability measure $\mathbb{P}_{x_{0}}^{(\varphi)}$, whose density with respect to $\mathbb{P}_{x_{0}}$, restricted to $\mathcal{F}_{n}$, is equal to $\varphi\left(X_{n \wedge \tau_{1}}\right)$ for all $n \geq 1$. 
Under $\mathbb{P}_{x_{0}}^{(\varphi)}$, the canonical process never returns to $x_{0}$, and more generally, to points where $\varphi$ vanishes: indeed, $\varphi\left(X_{n \wedge \tau_{1}}\right)=0$ when $\varphi\left(X_{n}\right)=0$, since we always have $\varphi\left(X_{\tau_{1}}\right)=\varphi\left(x_{0}\right)=0$. If $\left(x_{0}, x_{1}, \ldots, x_{n}\right)$ is such that $\varphi\left(x_{1}\right), \ldots, \varphi\left(x_{n}\right)$ are all strictly positive, we have for $n \geq 1$,

$$
\begin{aligned}
\mathbb{P}_{x_{0}}^{(\varphi)} & {\left[X_{0}=x_{0}, \ldots, X_{n}=x_{n}\right]=\varphi\left(x_{n}\right) \mathbb{P}_{x_{0}}\left[X_{0}=x_{0}, \ldots, X_{n}=x_{n}\right] } \\
& =\varphi\left(x_{n}\right) \prod_{j=1}^{n} p_{x_{j-1}, x_{j}}=\varphi\left(x_{1}\right) p_{x_{0}, x_{1}} \prod_{j=2}^{n}\left(\frac{\varphi\left(x_{j}\right)}{\varphi\left(x_{j-1}\right)} p_{x_{j-1}, x_{j}}\right)=\prod_{j=1}^{n} q_{x_{j-1}, x_{j}},
\end{aligned}
$$

where

and for all $x \neq x_{0}$,

$$
q_{x_{0}, y}=\varphi(y) p_{x_{0}, y},
$$

$$
q_{x, y}=\frac{\varphi(y)}{\varphi(x)} p_{x, y}
$$

with the essentially arbitrary convention $\varphi(y) / \varphi(x)=1$ in the case where $x \neq x_{0}$ and $\varphi(x)=0$. Since $\varphi$ is harmonic everywhere except at $x_{0}$, and $\sum_{y \in E} \varphi(y) p_{x_{0}, y}=$ 1 , the numbers $\left(q_{x, y}\right)_{x, y \in E}$ correspond to the transition probability of a Markov chain never returning to $x_{0}$. Hence, the canonical process under $\mathbb{P}_{x_{0}}^{(\varphi)}$ follows this chain, with starting point $x_{0}$.

We then have the following result, which may be seen as discrete version of the third equality from Salminen et al. (2015) cited at the beginning of the present paper:

Proposition 3.1. For all $n \geq 1$, the restriction of $\mathbf{p}_{n}$ to the trajectories such that $\varphi\left(X_{n}\right)>0$ is absolutely continuous with respect to the law of $\left(X_{0}, \ldots, X_{n}\right)$ under $\mathbb{P}_{x_{0}}^{(\varphi)}$, with density $1 / \varphi\left(X_{n}\right)$ (recall that $\varphi\left(X_{n}\right)>0$ almost surely under $\left.\mathbb{P}_{x_{0}}^{(\varphi)}\right)$.

Proof: The first measure is supported on the trajectories such that the values $\varphi\left(X_{1}\right), \varphi\left(X_{2}\right), \ldots, \varphi\left(X_{n}\right)$ are all strictly positive. Indeed, we already know that it is supported on the trajectories which do not return to $x_{0}$, and on the other hand, if $X_{j} \neq x_{0}$ and $\varphi\left(X_{j}\right)=0$, then $\varphi\left(X_{j+1}\right)=0$ almost surely under $\mathbb{P}_{x_{0}}$, by the harmonic property of $\varphi$, and then also under $\mathbf{p}_{n}$ if $j<n$. By induction, we deduce that $\varphi\left(X_{n}\right)=0$, which contradicts the fact that we restrict $\mathbf{p}_{n}$ to the paths such that $\varphi\left(X_{n}\right)>0$.

The measure with density $1 / \varphi\left(X_{n}\right)$ with respect to the law of $\left(X_{0}, \ldots, X_{n}\right)$ under $\mathbb{P}_{x_{0}}^{(\varphi)}$ is also supported on the trajectories such that $\varphi$ does not vanish except at time zero. Indeed, the transition probabilities $q_{x, y}$ of the Markov chain associated to $\mathbb{P}_{x_{0}}^{(\varphi)}$ vanish as soon as $\varphi(y)=0$, and $\varphi(x)>0$ or $x=x_{0}$.

Now, if $\left(x_{0}, \ldots, x_{n}\right)$ is a trajectory such that $\varphi\left(x_{j}\right)>0$ for all $j$ between 1 and $n$, we then quickly check that the two measures involved in the proposition give the same quantity, namely the product of $p_{x_{j-1}, x_{j}}$ for $j$ between 1 and $n$.

The following property of the measure $\mathbb{P}_{x_{0}}^{(\varphi)}$ is useful:

Proposition 3.2. The canonical process under $\mathbb{P}_{x_{0}}^{(\varphi)}$ is transient, i.e. it visits each state finitely many times.

Proof: Let $x$ be a state such that $\varphi(x)>0$. Since the initial recurrent Markov chain is irreducible and $\varphi\left(x_{0}\right)=0$, there exists $k>0$ minimal such that $\mathbb{P}_{x}\left[\varphi\left(X_{k}\right)=0\right]>$ 
0 . The harmonic properties of $\varphi$ then imply that $\mathbb{E}_{x}\left[\varphi\left(X_{k}\right)\right]=\varphi(x)$, since the chain a.s. does not hit $x_{0}$ before time $k$. Since

$$
\mathbb{P}_{x}\left[\varphi\left(X_{k}\right)<\varphi(x)\right] \geq \mathbb{P}_{x}\left[\varphi\left(X_{k}\right)=0\right]>0,
$$

we deduce that

$$
\mathbb{P}_{x}\left[\varphi\left(X_{k}\right)>\varphi(x)\right]>0 .
$$

Hence, there exist $y_{0}=x, y_{1}, \ldots, y_{k}$ such that $p_{y_{j-1}, y_{j}}>0$ for $0 \leq j \leq k, \varphi\left(y_{j}\right)>0$ for $1 \leq j \leq k-1$ (this fact is due to the minimality of $k$ ), and $\varphi\left(y_{k}\right)>\varphi(x)$. We have $q_{y_{j-1}, y_{j}}>0$, and then for all $n \geq 1$,

$$
\mathbb{P}_{x_{0}}^{(\varphi)}\left[X_{n+k}=y_{k} \mid X_{n}=x\right]>0 .
$$

By the strong Markov property, applied to the stopping times $\left(T_{p}\right)_{p \geq 1}$ given by

$$
T_{1}:=\inf \left\{n \geq 1, X_{n}=x\right\},
$$

and for $p \geq 1$,

$$
T_{p+1}:=\inf \left\{n \geq T_{p}+k+1, X_{n}=x\right\},
$$

we deduce that under $\mathbb{P}_{x_{0}}^{(\varphi)}$, the probability that the canonical process hits $x$ infinitely often without also hitting $y_{k}$ infinitely often is equal to zero.

We know that $\mathbb{P}_{x_{0}}^{(\varphi)}$-almost surely, $\varphi\left(X_{n}\right)>0$ for all $n \geq 1$. Moreover, $\left(1 / \varphi\left(X_{n}\right)\right)_{n \geq 1}$ is a supermartingale. Indeed, for $n \geq 1$, and $x \in E$ such that $\varphi(x)>0$,

$$
\mathbb{E}_{\mathbb{P}_{x_{0}}^{(\varphi)}}\left[1 / \varphi\left(X_{n+1}\right) \mid X_{n}=x\right]=\sum_{y \in E}(1 / \varphi(y)) \frac{\varphi(y)}{\varphi(x)} p_{x, y} \leq \frac{1}{\varphi(x)} \sum_{y \in E} p_{x, y}=\frac{1}{\varphi(x)} .
$$

Note that we don't have an equality in general since $(1 / \varphi(y)) \varphi(y)$ should be understood as 0 and not 1 if $\varphi(y)=0$ (which agrees with the usual convention $0 \times \infty=0$ in probability theory): indeed, in this case, the Markov chain following the distribution $\mathbb{P}_{x_{0}}^{(\varphi)}$ has probability transitions towards $y$ equal to zero, and then such a value of $y$ does not contribute to the sum corresponding to the expectation of $1 / \varphi\left(X_{n+1}\right)$ given $X_{n}=x$.

The supermartingale $\left(1 / \varphi\left(X_{n}\right)\right)_{n \geq 1}$ is positive, hence, it converges almost surely. If $\left(X_{n}\right)_{n \geq 1}$ visits some state $x$ infinitely often, necessarily with $\varphi(x)>0$, we deduce, from what we have seen above, that it almost surely also visits another state $y_{k}$ such that $\varphi\left(y_{k}\right)>\varphi(x)$. We deduce that $\left(1 / \varphi\left(X_{n}\right)\right)_{n>1}$ oscillates infinitely often between the distinct values $1 / \varphi(x)$ and $1 / \varphi\left(y_{k}\right)$, which can only happen with probability zero because of the a.s. convergence of the supermartingale.

We can look at two important examples: the simple symmetric random walk on $\mathbb{Z}$ and the simple symmetric random walk on $\mathbb{Z}^{2}$.

For the simple symmetric random walk on $\mathbb{Z}$, with $x_{0}=0$, the function $\varphi$ can be any convex combination of $x \mapsto 2 x_{+}$and $x \mapsto 2 x_{-}$, i.e. $\varphi(x)=\lambda x_{+}+\mu x_{-}$where $\lambda, \mu \geq 0, \lambda+\mu=2$. Here, the factor 2 is needed in order to have the normalization $\mathbb{E}_{x_{0}}\left[\varphi\left(X_{1}\right)\right]=1$.

In the case where $\varphi(x)=2 x_{+}$, the probability distribution $\mathbb{P}_{0}^{(\varphi)}$ corresponds to the Bessel random walk (which is a discrete analog of the Bessel process of dimension 3). 
For the simple symmetric random walk on $\mathbb{Z}^{2}$, with $x_{0}=(0,0)$, there is only one possibility for the function $\varphi$, given as follows:

$$
\varphi(x)=\lim _{N \rightarrow \infty}\left(\sum_{n=0}^{N} \mathbb{P}_{(0,0)}\left[X_{n}=(0,0)\right]-\sum_{n=0}^{N} \mathbb{P}_{x}\left[X_{n}=(0,0)\right]\right),
$$

where the limit can be shown to exist. One has in particular $\varphi(x)=1$ for $x \in \mathbb{Z}^{2}$ such that $\|x\|=1$, and for all $n \geq 1$,

$$
\varphi(n, n):=\varphi((n, n))=\frac{4}{\pi} \sum_{j=1}^{n} \frac{1}{2 j-1} .
$$

Moreover, for $x \neq(0,0)$,

$$
\varphi(x)=\frac{2}{\pi} \log \|x\|+\frac{2 \gamma_{\mathrm{Euler}}+\log 8}{\pi}+\mathcal{O}\left(\|x\|^{-2}\right),
$$

\|x $\mid$ denoting the Euclidean norm of $x$ (see Najnudel, 2015, pp. 289-290).

In these two examples, we can compute the probability that the Markov chain associated to $\mathbb{P}_{x_{0}}^{(\varphi)}$ does not return to $a$ after time $n$, conditionally on $X_{n}=a$, where $a \neq 0$. This computation will be used in the proof of the last proposition of the paper, which is related to the fourth equality stated at the beginning in the continuous setting. In the one-dimensional case, the computation of the probability just above is not difficult.

Proposition 3.3. Let us assume that we are in the setting of the simple random walk on $\mathbb{Z}$, and that $\varphi$ is a strict convex combination of $2 x_{+}$and $2 x_{-}$. If $a \neq x_{0}=0$, then we have, for any $n \geq 0$ such that $\mathbb{P}_{x_{0}}^{(\varphi)}\left(X_{n}=a\right)>0$ :

$$
\mathbb{P}_{x_{0}}^{(\varphi)}\left(\forall k>n, X_{k} \neq a \mid X_{n}=a\right)=\frac{1}{2|a|} .
$$

Proof: By the symmetry of the simple random walk on $\mathbb{Z}$, we can assume that $a>0$. In this case, conditionally on $X_{n}=a$, we have $X_{n+1}=a+1$ with probability $(a+1) / 2 a$ and $X_{n+1}=a-1$ with probability $(a-1) / 2 a$. These probabilities are computed by multiplying the transitions of the simple random walk by $\varphi\left(X_{n+1}\right) / \varphi\left(X_{n}\right)$. Then, we can further condition on $X_{n+1}$ :

- Conditionally on $X_{n+1}=a-1$, the Markov chain goes back to $a$ with probability one, since it tends to infinity.

- Conditionally on $X_{n+1}=a+1$, the process $\left(1 / X_{k}\right)_{k \geq n+1}$, stopped at the first time after $n+1$ when $X$ hits $a$, is a bounded martingale tending to zero if $X$ never returns to $a$ and to $1 / a$ if it returns to $a$. We deduce by the stopping theorem that the probability to never return to $a$ in this case is $1 /(a+1)$.

This gives the proposition, since

$$
\begin{aligned}
& \mathbb{P}_{x_{0}}^{(\varphi)}\left(\forall k>n, X_{k} \neq a \mid X_{n}=a\right) \\
& =\frac{a-1}{2 a} \mathbb{P}_{x_{0}}^{(\varphi)}\left(\forall k>n+1, X_{k} \neq a \mid X_{n+1}=a-1\right) \\
& +\frac{a+1}{2 a} \mathbb{P}_{x_{0}}^{(\varphi)}\left(\forall k>n+1, X_{k} \neq a \mid X_{n+1}=a+1\right) \\
& =\frac{a-1}{2 a} \cdot 0+\frac{a+1}{2 a} \cdot \frac{1}{a+1}=\frac{1}{2 a} .
\end{aligned}
$$


A similar formula holds in the two-dimensional case, but our proof is longer.

Proposition 3.4. In the setting of the two-dimensional simple random walk, with $x_{0}=(0,0)$, we have, for all $x, a, b \in \mathbb{Z}^{2}, a \neq b$,

$$
\mathbb{P}_{x}\left(T_{a}<T_{b}\right)=\frac{1}{2}\left(1+\frac{\varphi(x-b)-\varphi(x-a)}{\varphi(b-a)}\right),
$$

where for all $c \in \mathbb{Z}, T_{c}$ denotes the first hitting time of $c$, and for $a \neq 0$,

$$
\mathbb{P}_{a}\left(T_{(0,0)}<T_{a}^{\prime}\right)=1 /(2 \varphi(a)),
$$

where $T_{a}^{\prime}$ is the first return time at a. Finally for all $n \geq 1$,

$$
\mathbb{P}_{(0,0)}^{(\varphi)}\left(\forall k>0, X_{n+k} \neq a \mid X_{n}=a\right)=1 /(2 \varphi(a)) .
$$

Proof: We observe that the function $x \mapsto \mathbb{P}_{x}\left(T_{a}<T_{b}\right)$ is harmonic everywhere except at $a$ and $b$. This property remains true, for any $\lambda \in \mathbb{R}$, if we add $\lambda \varphi(x-a)$ to this function of $x$, since $x \mapsto \varphi(x-a)$ is harmonic everywhere except at $a$. Moreover, since $a \mapsto \varphi(x-a)$ is not harmonic at $a$, we have that

$$
\mathbb{P}_{a}\left(T_{a}<T_{b}\right)+\lambda \varphi(0)-\frac{1}{4} \sum_{v \in \mathbb{Z}^{2},\|v\|=1}\left(\mathbb{P}_{a+v}\left(T_{a}<T_{b}\right)+\lambda \varphi(v)\right)
$$

is a non-constant affine function of $\lambda$. Hence, there exists a value of $\lambda$ such that this quantity is equal to zero, and then

$$
x \mapsto \mathbb{P}_{x}\left(T_{a}<T_{b}\right)+\lambda \varphi(x-a),
$$

is harmonic at $a$, and then it is harmonic everywhere except at $b$ since we already know that it is harmonic on $\mathbb{Z}^{2} \backslash\{a, b\}$. Since $\varphi$ has logarithmic growth at infinity, the absolute value of the function just above is dominated by $1+\log (1+\|x\|)$. Hence, if the parameter $\mu$ is sufficiently large, we have that

$$
x \mapsto h(x):=\mathbb{P}_{x}\left(T_{a}<T_{b}\right)+\lambda \varphi(x-a)+\mu \varphi(x-b)
$$

is positive function, tending to infinity at infinity, and harmonic everywhere except at $b$. Let $M>0$ and let $S$ be the first hitting time of the union of $b$ and the complement in $\mathbb{Z}^{2}$ of the ball of center $b$ and radius $M$. For all $x \in \mathbb{Z}^{2},\left(h\left(X_{n \wedge S}\right)\right)_{n \geq 0}$ is a bounded martingale, since $h$ is harmonic on $\mathbb{Z}^{2} \backslash\{b\}$, and then, by letting $n \rightarrow \infty$, we deduce that $\mathbb{E}\left[h\left(X_{S}\right)\right]=h(x)$. Now, since $h$ tends to infinity at infinity, we have $h \geq h(b)$ on the complement of the ball of center $b$ and radius $M$, as soon as $M$ is large enough. Hence, $h\left(X_{S}\right) \geq h(b)$ almost surely, which shows that $h(x) \geq h(b)$. Therefore, $h-h(b)$ is a nonnegative function, equal to zero at $b$, and harmonic everywhere else. By the uniqueness property satisfied by $\varphi$, we deduce that $h(x)-h(b)$ is a multiple of $\varphi(x-b)$, and then

$$
\mathbb{P}_{x}\left(T_{a}<T_{b}\right)=c_{1}+c_{2} \varphi(x-a)+c_{3} \varphi(x-b)
$$

for three constants $c_{1}, c_{2}, c_{3}$. By looking at the behavior at infinity, $c_{2}=-c_{3}$. Taking $x=a$ and $x=b$ then gives

$$
\begin{aligned}
& 1=c_{1}-c_{2} \varphi(a-b), \\
& 0=c_{1}+c_{2} \varphi(b-a),
\end{aligned}
$$

and then $c_{1}=1 / 2, c_{2}=-1 /(2 \varphi(b-a))$, since $\varphi(a-b)=\varphi(b-a)$ by symmetry. This gives the first part of the proposition. 
The second part of the proposition is obtained by observing that if $c$ is a neighbor of $a$,

$$
\mathbb{P}_{c}\left(T_{0}<T_{a}\right)=\frac{1}{2}\left(1+\frac{\varphi(c-a)-\varphi(c)}{\varphi(a)}\right)=\frac{1}{2}\left(1+\frac{1-\varphi(c)}{\varphi(a)}\right) .
$$

Taking the average with the four neighbors of $a$, and observing that the average of $\varphi$ on these neighbors is equal to $\varphi(a)$ since $\varphi$ is harmonic at $a \neq 0$, we get

$$
\mathbb{P}_{a}\left(T_{0}<T_{a}^{\prime}\right)=\frac{1}{2}\left(1+\frac{1-\varphi(a)}{\varphi(a)}\right)=\frac{1}{2 \varphi(a)} .
$$

For the last computation, we observe, for $p \geq 1$,

$$
\begin{gathered}
\mathbb{P}_{(0,0)}^{(\varphi)}\left(X_{n+1}, \ldots, X_{n+p-1} \neq a, X_{n+p}=a \mid X_{n}=a\right) \\
=\sum_{r_{1}, \ldots, r_{p-1} \neq a} q_{a, r_{1}} q_{r_{1}, r_{2}} \ldots q_{r_{p-1}, a},
\end{gathered}
$$

where $q$ corresponds to the transition probabilities of the Markov chain associated to $\mathbb{P}_{(0,0)}^{(\varphi)}$. If $p$ denotes the transitions of the standard random walk, we can write $q$ in function of $p$ and this gives, after simplifying a telescopic product involving $\varphi$ (observe that the trajectories here start and stop at the same point $a$ ):

$$
\begin{gathered}
\mathbb{P}_{(0,0)}^{(\varphi)}\left(X_{n+1}, \ldots, X_{n+p-1} \neq a, X_{n+p}=a \mid X_{n}=a\right) \\
=\sum_{r_{1}, \ldots, r_{p-1} \neq a,(0,0)} p_{a, r_{1}} p_{r_{1}, r_{2}} \ldots p_{r_{p-1}, a},
\end{gathered}
$$

and then

$$
\begin{gathered}
\mathbb{P}_{(0,0)}^{(\varphi)}\left(X_{n+1}, \ldots, X_{n+p-1} \neq a, X_{n+p}=a \mid X_{n}=a\right) \\
\quad=\mathbb{P}_{a}\left(X_{1}, \ldots, X_{p-1} \neq a,(0,0), X_{p}=a\right)
\end{gathered}
$$

which gives

$$
\mathbb{P}_{(0,0)}^{(\varphi)}\left(X_{n+1}, \ldots, X_{n+p-1} \neq a, X_{n+p}=a \mid X_{n}=a\right)=\mathbb{P}_{a}\left(T_{(0,0)}>T_{a}^{\prime}=p\right) .
$$

Adding for all $p \geq 1$, we get

$$
\mathbb{P}_{(0,0)}^{(\varphi)}\left(\exists k, X_{n+k}=a \mid X_{n}=a\right)=\mathbb{P}_{a}\left(T_{(0,0)}>T_{a}^{\prime}\right),
$$

and then

$$
\mathbb{P}_{(0,0)}^{(\varphi)}\left(\forall k, X_{n+k} \neq a \mid X_{n}=a\right)=\mathbb{P}_{a}\left(T_{(0,0)}<T_{a}^{\prime}\right)=\frac{1}{2 \varphi(a)}
$$

Remark 3.5. Proposition 3.4 can also deduced from a more general discussion on recurrent two-dimensional random walks given in Chapter III of the book by Spitzer Spitzer (1976) (see in particular Section 11). A particular case of this result is the following: the probability that the simple random walk starting at $(0,0)$ reaches $(0,1)$ before returning to $(0,0)$ is equal to $1 / 2$.

In the cases of simple random walk on $\mathbb{Z}$ or $\mathbb{Z}^{2}$, we have the following relation between the excursions and the measure $\mathbb{P}_{x_{0}}^{(\varphi)}$, which corresponds to the fourth equality from Salminen et al. (2015) stated at the beginning of the present paper: 
Proposition 3.6. Let us assume that we are in the setting of the simple random walk on $\mathbb{Z}$ or $\mathbb{Z}^{2}$, with $x_{0}$ equal to the origin. Moreover, in the one-dimensional case, let us assume that $\varphi(x)=|x|$. We recall that there is only one possible function $\varphi$ in the two-dimensional case. Under these assumptions, we have:

$$
\sum_{n=1}^{\infty} \mathbf{p}_{n}=2 \sum_{b \in E \backslash\left\{x_{0}\right\}} \mathbb{P}_{x_{0}}^{(\varphi), \gamma_{b}}
$$

where $\mathbb{P}_{x_{0}}^{(\varphi), \gamma_{b}}$ is the law of the canonical trajectory, stopped at its last hitting time of $b$ under the restriction of $\mathbb{P}_{x_{0}}^{(\varphi)}$ to the trajectories hitting $b$ at least once.

Proof: All the trajectories with positive measure under the two sides of the equality hit $x_{0}$ only at time 0 . Let $\left(x_{0}, \ldots, x_{n}\right)$ be such a trajectory, for $n \geq 1$. Under the left-hand side, or equivalently, under $\mathbf{p}_{n}$, its measure is the product of $p_{x_{j-1}, x_{j}}$ for $j$ between 1 and $n$. Under the right-hand side, or equivalently, under $2 \mathbb{P}_{x_{0}}^{(\varphi), \gamma_{x_{n}}}$, the measure is twice the product of $q_{x_{j-1}, x_{j}}$ for $1 \leq j \leq n$, multiplied by the probability, under $\mathbb{P}_{x_{0}}^{(\varphi)}$, and conditionally on $X_{n}=x_{n}$, that the canonical trajectory does not hit $x_{n}$ again after time $n$. Hence, the measure is equal to

$$
2 \varphi\left(x_{1}\right) p_{x_{0}, x_{1}} \prod_{j=2}^{n}\left(\frac{\varphi\left(x_{j}\right)}{\varphi\left(x_{j-1}\right)} p_{x_{j-1}, x_{j}}\right)\left(\frac{1}{2 \varphi\left(x_{n}\right)}\right)=\frac{1}{2} \prod_{j=1}^{n} p_{x_{j-1}, x_{j}} .
$$

\section{References}

$\mathrm{Ph}$. Biane and M. Yor. Valeurs principales associées aux temps locaux browniens. Bull. Sci. Math. (2) 111 (1), 23-101 (1987). MR886959.

J. Najnudel. On $\sigma$-finite measures related to the Martin boundary of recurrent Markov chains. In In memoriam Marc Yor-Séminaire de Probabilités XLVII, volume 2137 of Lecture Notes in Math., pages 263-297. Springer, Cham (2015). MR3444303.

J. Najnudel, B. Roynette and M. Yor. A global view of Brownian penalisations, volume 19 of MSJ Memoirs. Mathematical Society of Japan, Tokyo (2009). ISBN 978-4-931469-52-5. MR2528440.

P. Salminen, P. Vallois and M. Yor. On the excursion theory for linear diffusions. Jpn. J. Math. 2 (1), 97-127 (2007). MR2295612.

P. Salminen, J.-Y. Yen and M. Yor. Integral representations of certain measures in the one-dimensional diffusions excursion theory. In In memoriam Marc YorSéminaire de Probabilités XLVII, volume 2137 of Lecture Notes in Math., pages 1-15. Springer, Cham (2015). MR3444291.

F. Spitzer. Principles of random walk. Springer-Verlag, New York-Heidelberg, second edition (1976). MR0388547. 